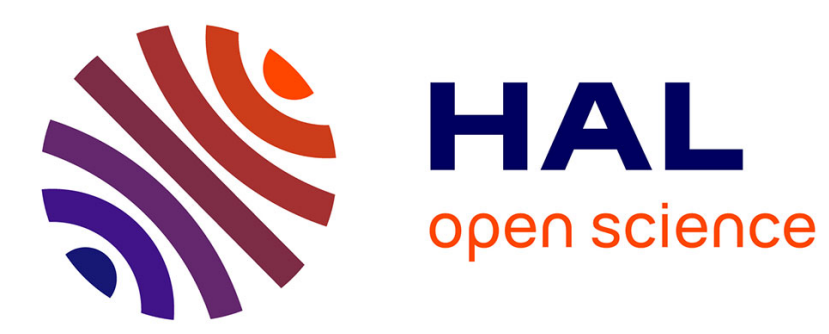

\title{
Weakly one-based geometric theories
}

Alexander Berenstein, Evgueni Vassiliev

\section{To cite this version:}

Alexander Berenstein, Evgueni Vassiliev. Weakly one-based geometric theories. 2010. hal-00488140

\section{HAL Id: hal-00488140 \\ https://hal.science/hal-00488140}

Preprint submitted on 1 Jun 2010

HAL is a multi-disciplinary open access archive for the deposit and dissemination of scientific research documents, whether they are published or not. The documents may come from teaching and research institutions in France or abroad, or from public or private research centers.
L'archive ouverte pluridisciplinaire HAL, est destinée au dépôt et à la diffusion de documents scientifiques de niveau recherche, publiés ou non, émanant des établissements d'enseignement et de recherche français ou étrangers, des laboratoires publics ou privés. 


\title{
WEAKLY ONE-BASED GEOMETRIC THEORIES
}

\author{
ALEXANDER BERENSTEIN AND EVGUENI VASSILIEV
}

\begin{abstract}
We study the class of weakly locally modular geometric theories introduced in [5], a common generalization of the classes of linear SU-rank 1 and linear o-minimal theories. We find new conditions equivalent to weak local modularity: weak one-basedness and the absence of type definable almost quasidesigns. Among other things, we show that weak one-basedness is closed under reducts and generic predicate expansions. We also show that a lovely pair expansion of a non-trivial weakly one-based $\omega$-categorical superrosy thorn rank 1 theory interprets an infinite vector space over a finite field.
\end{abstract}

\section{INTRODUCTION}

It is a well known fact [21] that for a strongly minimal theory $T$, the following conditions are equivalent: i) $T$ is linear, ii) $T$ is 1-based, iii) $T$ is locally modular. Furthermore, these conditions are preserved under reducts. For a simple $S U$-rank one theory $T$ the picture changes slightly, it is proved in $[23,5]$ that for such a theory $T$, it is equivalent that: i) $T$ is 1-based, ii) $T$ is linear and iii) $T$ is weakly locally modular (see Definition 2.1). It is also known (e.g. see [23]) that in the $S U$-rank one setting local modularity is a strictly stronger condition than being 1based. A more general framework where we can still study the geometry associated to the algebraic closure is the class of geometric theories. Recall that a geometric theory is a complete theory $T$ such that for any model $M \models T$, the algebraic closure satisfies the Exchange Property and in addition $T$ eliminates the quantifier $\exists^{\infty}$. Examples include strongly minimal theories, simple $S U$ rank 1 theories, dense o-minimal theories and the theory of the $p$-adics. Inside a model of a geometric theory, algebraic independence gives a good notion of independence for real tuples. A key example of the behavior of linearity in o-minimal theories is the following theory first introduced in [15].

Example 1.1. Let $\mathcal{R}=\left(\mathbb{R},+,<,\left.f\right|_{(-1,1)}\right)$ where $f$ is defined by $f(x)=\pi x$. Clearly, $\left.f\right|_{(-1,1)}$ can be extended to all of $\mathcal{R}$ by $f(x)=n f\left(\frac{x}{n}\right)$ for $x \in(-n, n)$, however this extension is not uniformly definable, and thus in a sufficiently saturated model $\mathcal{R}^{*}$ of $T=T h(\mathcal{R})$, we cannot define $f(x)$ for "infinite" elements. As the theory of a reduct of a vector space over $\mathbb{Q}(\pi), T$ is a linear $(\mathrm{CF})$ theory, but it is not locally modular. It is also shown in [21] that $T$ does not have almost

2000 Mathematics Subject Classification. 03C45, 03C64.

Key words and phrases. geometric structures, linear structures, rosy theories, geometries, lovely pairs.

The first author was supported by a grant from Facultad de Ciencias, Universidad de los Andes and by the ANR chaire d'excellence junior THEMODMET (ANR-06-CEXC- 007) while visiting Université Claude Bernard Lyon 1 during the academic year 2008-2009; the second author was supported by a NSERC grant. 
canonical bases, i.e. a smallest algebraically closed subset over which a type is free. The theory $T$ also fails to be 1-based, i.e. there is $M \models T$ saturated and there are sets $A, B \subset M$ such that $A \bigotimes_{\mathrm{acl}^{e q}(A) \cap \operatorname{acl}^{e q}(B)} B$.

The example above shows that inside a geometric theory $T$, local modularity and 1-basedness do not need to be preserved under reducts. The main reason for the failure of the second condition is the absence of almost canonical bases in the reduct.

The origin of the expression 1-basedness comes from the concept that in a 1-based simple theory one element in a Morley sequence contains all the information about the original type (in general we require a countable Morley sequence to recover all the information). Following this idea we introduce the notion of weak 1-basedness (see Definition 2.3), prove that this notion coincides, in the setting of geometric structures, with the notion weak local modularity introduced in [5] (see Definition 2.1 ) and finally show that it is preserved under reducts.

The main goal of this paper is to study the class of weakly 1-based geometric structures. All linear o-minimal theories, including the one presented in Example 1.1, as well as linear $S U$ rank 1 theories are examples of weakly 1-based theories.

Our work is divided as follows:

In the second section of this paper we define weak local modularity, weak 1basedness and show that the notions coincide. We also introduce the notion of type definable almost quasidesigns, prove that it coincides with weak local modularity and use it to show that a reduct of a weakly locally modular theory is again weakly locally modular.

In section three we study the geometry associated to a weakly 1-based geometric theory. We follow the approach from [23] and show that a lovely pair associated to a non-trivial weakly 1-based $\omega$-categorical superrosy thorn rank 1 theory interprets an infinite vector space over a finite field.

In section four we generalize the notion of weak 1-basedness to the setting of rosy theories. We show that, under some mild assumptions, if $T$ is a thorn rank one rosy weakly 1-based theory, then the associated theory $T_{P}$ of lovely pairs of $T$ is again weakly 1-based.

In section five we concentrate on examples: we show that the expansion of a weakly 1-based theory with a generic predicate is again weakly 1-based and prove that divisible groups with the Mann property inside a real closed field with the induced structure from the field are also weakly 1-based. Finally, in the last section, we show that the dense embeddings studied by Macintyre in [16] are a special case of lovely pairs of geometric structures.

We assume that the reader is familiar with the results on lovely pairs of geometric structures presented in [5] (although no familiarity with lovely pairs is needed for most of section 2 and section 5). We will now recall the definition and basic properties of lovely pairs.

Definition 1.2. We say that an elementary pair of models $P(M) \preceq M$ of a geometric theory $T$ is a lovely pair of models of $T$ if

(1) (density/coheir property) if $A \subset M$ is algebraically closed and finite dimensional and $q \in S_{1}(A)$ is non-algebraic, then there is $a \in P(M)$ such that $a \models q$; 
(2) (extension property) if $A \subset M$ is algebraically closed and finite dimensional and $q \in S_{1}(A)$ is non-algebraic, then there is $a \in M, a \models q$ and $a \notin$ $\operatorname{acl}(A \cup P(M))$.

Any elementary pair of models extends to a lovely one. Any two lovely pairs of models of a geometric theory are elementarily equivalent, thus giving rise to a complete theory $T_{P}$ in the expanded language $L_{P}=L(T) \cup\{P\}$. The class of lovely pairs of models of $T$ is almost an elementary class: sufficiently saturated models of $T_{P}$ are again lovely pairs.

Lovely pairs of geometric structures are a common generalization of lovely pairs of supersimple SU-rank 1 structures [23] and (sufficiently saturated) dense pairs of o-minimal expansions of ordered abelian groups [10].

Given a pair $(M, P)$ and a set $A \subset M$, we say that $A$ is $P$-independent, if $A \downarrow_{P(A)} P(M)$ where $P(A)=A \cap P(M)$. Any two $P$-independent tuples $\vec{a}$ and $\vec{b}$ in a lovely pair, satisfying the same quantifier free $L_{P}$-type, have the same $L_{P}$-type.

When working in lovely pairs, we will refer to the operator

$$
\operatorname{scl}(-)=\operatorname{acl}(-\cup P(M))
$$

as the small closure. Note that a small closure of any set is algebraically closed in the sense of $T_{P}$. We write $\operatorname{tp}_{P}$ and $\operatorname{acl}_{P}$ for types and algebraic closure in the sense of $T_{P}$.

The following is a result of Boxall [6] (generalizing a fact from [23] to the setting of superrosy theories of p-rank 1):

Fact 1.3. Suppose $T$ is a p-rank 1 theory that eliminates $\exists^{\infty}$. Then $T_{P}$ is superrosy of $p$-rank $\leq \omega$. Moreover:

(1) Any definable "large" set in a lovely pair $(M, P)$ (i.e. a set definable over $A$ such that it has a realization in $M \backslash \operatorname{acl}(P(M) \cup A)$ ) does not p-divide over $\emptyset$.

(2) Any infinite definable subset of $P(M)$ does not $p$-divide over $\emptyset$. In particular, $P(M)$ has p-rank 1 in $(M, P)$.

Thus, when $T$ is rosy of thorn rank $1, T_{P}$ is again super-rosy; we write $\downarrow^{P}$ for thorn independence in models of $T_{P}$.

\section{WEAK LOCAL MODULARITY, WEAK 1-BASEDNESS AND LINEARITY}

Our goal in this section is to study, in the setting of geometric theories, analogues to the notions of local modularity, 1-basedness and linearity that are well understood in the setting of minimal stable theories [20] and $S U$-rank one simple theories [23].

In [5] we studied a notion called weak local modularity using lovely pairs of structures and provided several characterizations of it. We recall the definition:

Definition 2.1. (See [5, Theorem 1]) Let $T$ be a geometric theory. We say that $T$ is weakly locally modular if for $\mathcal{M} \models T$ saturated and $A, B \subset M$ there exist $C \downarrow A B$ such that $A \downarrow_{\operatorname{acl}(A C) \cap \operatorname{acl}(B C)} B$

In [5] we showed this notion coincided with 1-basedness for $S U$-rank one simple theories and with linearity for o-minimal theories. We have also shown that weak local modularity is equivalent to $\operatorname{acl}_{P}=$ acl and to modularity of scl in models of $T_{P}$. We also proved: 
Fact 2.2. ([5, Proposition 4.8]) If $T$ is a weakly locally modular superrosy geometric theory of p-rank 1 , then $T_{P}$ has p-rank $\leq 2$.

Note that in the SU-rank 1 case [23], weak local modularity of $T$ is actually equivalent to SU-rank of $T$ being $\leq 2$.

We now introduce a notion that is an analogue of 1-basedness in the setting of geometric theories.

Definition 2.3. Let $T$ be a geometric theory. We say that $T$ is weakly 1 -based if whenever $M \models T$ is saturated, $\vec{a} \in M$ and $B \subset M$, there is $\vec{a}^{\prime} \models \operatorname{tp}(\vec{a} / B)$ independent from $\vec{a}$ over $B$, such that $\vec{a} \downarrow_{\vec{a}^{\prime}} B$.

In the stable or simple setting, a rank one theory is locally modular if and only if it is 1-based. The proof uses the notion of canonical bases. An analogue of his notion can be defined in the setting of geometric structures, see for example [21]:

Definition 2.4. Let $T$ be a geometric theory and let $M \models T$ be saturated. We say $T$ has almost canonical bases if whenever $A \subset M$ is algebraically closed and $a_{1}, \ldots, a_{n} \in M$, there is a smallest $B \subset A$ algebraically closed such that $\operatorname{tp}\left(a_{1}, \ldots, a_{n} / A\right)$ is free over $B$.

The main problem with this notion is that almost canonical bases need not exist in geometric structures (see [21] and Example 1.1). When they exists, the proofs in [23] that show the equivalence of local modularity and 1-basedness for $S U$-rank one simple theories can be used almost word-by-word to prove the equivalence of weak local modularity and weak 1-basedness in the setting of geometric theories. Instead, we will show that weak local modularity agrees with weak 1-basedness using stronger formulations of weak 1-basedness.

We start with a technical lemma.

Lemma 2.5. Let $T$ be a geometric theory and let $\mathcal{M} \models T$ be saturated. Let $\vec{a} \in M$, $B \subset M$ and $\vec{a}^{\prime} \in M$ be such that $\operatorname{tp}(\vec{a} / B)=\operatorname{tp}\left(\vec{a}^{\prime} / B\right), \vec{a} \downarrow_{B} \vec{a}^{\prime}$ and $\vec{a} \downarrow_{\vec{a}^{\prime}} B$. Then $\vec{a}^{\prime} \downarrow_{\vec{a}} B$.

Proof. We can write $\vec{a}=\vec{a}_{1} \vec{a}_{2}$, where $\vec{a}_{1}$ is an independent tuple over $B$ and $\vec{a}_{2} \in$ $\operatorname{acl}\left(\vec{a}_{1}, B\right)$. In the same way write $\vec{a}^{\prime}=\vec{a}_{1}^{\prime} \vec{a}_{2}^{\prime}$ with $\operatorname{tp}\left(\vec{a}_{1}, \vec{a}_{2} / B\right)=\operatorname{tp}\left(\vec{a}_{1}^{\prime}, \vec{a}_{2}^{\prime} / B\right)$. Note that $\operatorname{dim}\left(\vec{a}_{1} \vec{a}_{2} \vec{a}_{1}^{\prime} \vec{a}_{2}^{\prime}\right)=\operatorname{dim}\left(\vec{a}_{1} \vec{a}_{2}\right)+\operatorname{dim}\left(\vec{a}_{1}^{\prime} \vec{a}_{2}^{\prime} / \vec{a}_{1} \vec{a}_{2}\right)=\operatorname{dim}\left(\vec{a}_{1}^{\prime} \vec{a}_{2}^{\prime}\right)+\operatorname{dim}\left(\vec{a}_{1} \vec{a}_{2} / \vec{a}_{1}^{\prime} \vec{a}_{2}^{\prime}\right)$, so $\operatorname{dim}\left(\vec{a}^{\prime} / \vec{a}\right)=\operatorname{dim}\left(\vec{a} / \vec{a}^{\prime}\right)=\operatorname{dim}\left(\vec{a} / B \vec{a}^{\prime}\right)=\operatorname{dim}(\vec{a} / B)=\left|\vec{a}_{1}\right|=\left|\vec{a}_{1}^{\prime}\right|$. Thus $\operatorname{dim}\left(\vec{a}^{\prime} / \vec{a}\right)=\left|\vec{a}_{1}^{\prime}\right|=\operatorname{dim}\left(\vec{a}^{\prime} / B \vec{a}\right)$ and $\vec{a}^{\prime} \downarrow_{\vec{a}} B$.

Proposition 2.6. Let $T$ be a geometric theory and let $\mathcal{M} \models T$ be saturated. Then the following conditions are equivalent:

(1) $T$ is weakly 1-based.

(2) Whenever $\vec{a} \in M, B \subset M$, there is $C \downarrow_{B} \vec{a}$ such that for all $\vec{a}^{\prime} \models$ $\operatorname{tp}(\vec{a} / \operatorname{acl}(B C))$ independent from $\vec{a}$ over $B C$, we have $\vec{a} \downarrow_{\vec{a}^{\prime}} B$.

(3) Whenever $\vec{a} \in M, B \subset M$, there is $C \downarrow_{B} \vec{a}$ such that for all $\vec{a}^{\prime} \models$ $\operatorname{tp}(\vec{a} / \operatorname{acl}(B C))$ independent from $\vec{a}$ over $B C$, we have $\vec{a} \downarrow_{\vec{a}^{\prime}} B C$.

Proof. (1) $\Longrightarrow(2)$. Let $\vec{a} \in M$ and $B \subset M$. Since $T$ is weakly 1-based, there exists $\vec{a}^{\prime}=\operatorname{tp}(\vec{a} / B)$ such that $\vec{a}^{\prime} \downarrow_{B} \vec{a}$ and $\vec{a}^{\prime} \downarrow_{\vec{a}} B$. Let $C=\vec{a}_{1}^{\prime}$.

Claim Whenever $\vec{c}=\operatorname{tp}(\vec{a} / \operatorname{acl}(B C))$ is independent from $\vec{a}$ over $B C$, we have $\vec{a}$ is independent from $B$ over $\vec{c}$. 
Let $\vec{c}=\operatorname{tp}(\vec{a} / \operatorname{acl}(B C))$ be such that $\vec{c} \downarrow_{B C} \vec{a}$. Since $\vec{a} \downarrow_{\vec{a}^{\prime}} B$, we have $\vec{c} B \downarrow_{\vec{a}^{\prime}} \vec{a}$ and thus

$$
B \underset{\vec{a}^{\prime} \vec{c}}{\downarrow} \vec{a} \quad(*)
$$

Since $\vec{a} \downarrow_{\vec{a}^{\prime}} B$ and $\vec{c}=\operatorname{tp}(\vec{a} / B C)$ we get $\vec{c} \downarrow_{\vec{a}^{\prime}} B$. Using Lemma 2.5 this implies $\vec{a}^{\prime} \downarrow_{\vec{c}} B$ and together with (*) we get $B \downarrow_{\vec{c}} \vec{a}$.

$(2) \Longrightarrow(3)$. This direction is mostly forking calculus. With the assumptions from (3), we can show using condition (2) that $\vec{a} \downarrow_{\vec{a}^{\prime}} B$. On the other hand, we have $\vec{a} \downarrow_{B C} \vec{a}^{\prime}$ and $\vec{a} \downarrow_{B} C$, so by transitivity $\vec{a} \downarrow_{B} C \vec{a}^{\prime}$ and thus $\vec{a} \downarrow_{B \vec{a}^{\prime}} B C$. This fact together with $\vec{a} \downarrow_{\vec{a}^{\prime}} B$ gives us $\vec{a} \downarrow_{\vec{a}^{\prime}} B C$ as desired.

$(3) \Longrightarrow(1)$ is clear.

Theorem 2.7. Let $T$ be a geometric theory. Then the following conditions are equivalent:

(1) $T$ is weakly 1-based.

(2) $T$ is weakly locally modular.

Proof. (2) $\Longrightarrow$ (1). Let $\mathcal{M} \models T$ be saturated. Let $\vec{a}_{1}, \vec{a}_{1} \in M$ and $B \subset M$ be such that $\vec{a}_{1}$ is an independent tuple over $B$ and $\vec{a}_{2} \in \operatorname{acl}\left(B, \vec{a}_{1}\right)$. Since $T$ is weakly locally modular, there exists $C \downarrow \vec{a}_{1} \vec{a}_{2} B$ such that $\vec{a}_{1} \vec{a}_{2} \downarrow_{\text {acl }\left(\vec{a}_{1} \vec{a}_{2} C\right) \cap \operatorname{acl}(B C)} B C$

Let $\vec{a}_{1}^{\prime} \vec{a}_{2}^{\prime}=\operatorname{tp}\left(\vec{a}_{1} \vec{a}_{2} / \operatorname{acl}(B C)\right)$ be independent from $\vec{a}_{1} \vec{a}_{2}$ over acl $(B C)$. Then $\operatorname{acl}\left(\vec{a}_{1} \vec{a}_{2} C\right) \cap \operatorname{acl}(B C)=\operatorname{acl}\left(\vec{a}_{1}^{\prime} \vec{a}_{2}^{\prime} C\right) \cap \operatorname{acl}(B C)$, so $\vec{a}_{1} \vec{a}_{2} \downarrow_{\operatorname{acl}\left(\vec{a}_{1}^{\prime} \vec{a}_{2}^{\prime} C\right) \cap \operatorname{acl}(B C)} B C$. It is also clear that $\vec{a}_{1} \vec{a}_{2} \downarrow_{B C} \vec{a}_{1}^{\prime} \vec{a}_{2}^{\prime} B C$. Thus

$$
\operatorname{tp}\left(\vec{a}_{1} \vec{a}_{2} / \operatorname{acl}\left(\vec{a}_{1}^{\prime} \vec{a}_{2}^{\prime} C\right) \cap \operatorname{acl}(B C)\right) \subset \operatorname{tp}\left(\vec{a}_{1} \vec{a}_{2} / \operatorname{acl}(B C)\right) \subset \operatorname{tp}\left(\vec{a}_{1} \vec{a}_{2} / \operatorname{acl}\left(\vec{a}_{1}^{\prime} \vec{a}_{2}^{\prime} B C\right)\right)
$$

is a chain of free extensions, so $\vec{a}_{1} \vec{a}_{2} \downarrow_{\operatorname{acl}\left(\vec{a}_{1}^{\prime} \vec{a}_{2}^{\prime} C\right) \cap \operatorname{acl}(B C)} \vec{a}_{1}^{\prime} \vec{a}_{2}^{\prime} B C$ and thus $\vec{a}_{1} \vec{a}_{2} \downarrow_{\vec{a}_{1}^{\prime} \vec{a}_{2}^{\prime} C} B$. On the other hand, since $C \downarrow \vec{a}_{1}^{\prime} \vec{a}_{2}^{\prime} B$, we have $\vec{a}_{1}^{\prime} \vec{a}_{2}^{\prime} C \downarrow_{\vec{a}_{1}^{\prime} \vec{a}_{2}^{\prime}} \vec{a}_{1}^{\prime} \vec{a}_{2}^{\prime} B$, and by symmetry and transitivity of independence $\vec{a}_{1} \vec{a}_{2} \downarrow_{\vec{a}_{1}^{\prime} \vec{a}_{2}^{\prime}} B$ as we wanted.

(1) $\Longrightarrow(2)$. Let $\mathcal{M}=T$ be saturated. Let $\vec{a}, \vec{b} \in M$ and $B \subset M$ be such that $\vec{a}$ is an independent tuple over $B$ and $\vec{b} \in \operatorname{acl}(B, \vec{a})$. Since $T$ is weakly 1-based there exists $\vec{a}^{\prime} \vec{b}^{\prime} \models \operatorname{tp}(\vec{a} \vec{b} / B)$ such that $\vec{a} \vec{b} \downarrow_{B} \vec{a}^{\prime} \vec{b}^{\prime}$ and $\vec{a} \vec{b} \downarrow_{\vec{a}^{\prime} \vec{b}^{\prime}} B$. Let $C=\vec{a}^{\prime}$, notice that $C \downarrow B \vec{a} \vec{b}$ and $\vec{a} \vec{b} \downarrow_{\vec{a}^{\prime} b^{\prime}} B C$.

Claim $\vec{a} \vec{b} \downarrow_{\operatorname{acl}(\vec{a} \vec{b} C) \cap \operatorname{acl}(B C)} B$.

First note that $\vec{b}^{\prime} \in \operatorname{acl}\left(B \vec{a}^{\prime}\right)$. Since $\vec{a} \vec{b} \downarrow_{\vec{a}^{\prime} \vec{b}^{\prime}} B$ by Lemma 2.5 we have $\vec{a}^{\prime} \vec{b}^{\prime} \downarrow_{\vec{a} \vec{b}} B$ and thus $\vec{b}^{\prime} \in \operatorname{acl}\left(\vec{a}^{\prime} \vec{a} \vec{b}\right)$. Thus $\vec{a}^{\prime} \vec{b}^{\prime} \in \operatorname{acl}(\vec{a} \vec{b} C) \cap \operatorname{acl}(B C)$ and we get $\vec{a} \vec{b} \downarrow_{\operatorname{acl}(\vec{a} \vec{b} C) \cap \operatorname{acl}(B C)} B$ as desired.

Remark 2.8. By Theorem 4.3, [5], in the definition of weak local modularity we can assume that one of the two sets is in fact a 2-tuple, i.e. we require that for any $a b$ and a set $B$ such that $a \in \operatorname{acl}(B b)$, there exists a $C \downarrow_{\emptyset} B a b$ such that $a \in \operatorname{acl}(C b d)$ for some $d \in \operatorname{acl}(B C)$. Therefore in the proof of $((1) \Longrightarrow(2))$ above we can assume that $\vec{a}$ and $\vec{b}$ are 1-tuples. Thus in the definition of weak 1-basedness and in the conditions (2) and (3) in 2.6 we may assume that $\vec{a}$ is a 2 -tuple. 
Now we will connect weak 1-basedness with the notion of quasidesign. It is well-known that a stable theory is 1-based if and only if $T$ has no complete-typedefinable quasidesign (see [20]). In our setting, we need to introduce the following modification.

Definition 2.9. We say that a partial type $r(\vec{x}, \vec{y})$ over a set $A$ defines a partial almost quasidesign, if

(1) there are $\vec{b}, \vec{c}$ such that $=r(\vec{b}, \vec{c}), \vec{b} \notin \operatorname{acl}(\vec{c}, A)$ and $\vec{c} \notin \operatorname{acl}(\vec{b}, A)$;

(2) whenever $\vec{c} \notin \operatorname{acl}\left(\overrightarrow{c^{\prime}}, A\right)$ and $\overrightarrow{c^{\prime}} \notin \operatorname{acl}(\vec{c}, A), r(\vec{x}, \vec{c}) \wedge r\left(\vec{x}, \overrightarrow{c^{\prime}}\right)$ is finite.

If $r$ is complete, we refer to such partial quasidesign as complete. In the complete case, we can replace "there are" by "for any" in (1). Clearly, any partial quasidesign gives rise to a complete one, if we take $\operatorname{tp}(\vec{b} \vec{c} / A)$ where $\vec{b}$ and $\vec{c}$ come from (1).

Proposition 2.10. The following are equivalent for any geometric theory $T$.

(1) $T$ is weakly 1-based

(2) $T$ does not have a partial almost quasidesign

(3) $T$ does not have a complete almost quasidesign

Proof. $(1 \rightarrow 2)$ Suppose $T$ is weakly 1 -based, and $r(\vec{x}, \vec{y})$ defines a partial almost quasidesign. Adding the parameters of $r$ to the language, we may assume that $r$ is defined over $\emptyset$. Take $\vec{b}$ and $\vec{c}$ such that $=r(\vec{b}, \vec{c}), \vec{b} \notin \operatorname{acl}(\vec{c})$ and $\vec{c} \notin \operatorname{acl}(\vec{b})$. By weak 1-basedness we can find $\overrightarrow{c^{\prime}} \mid=\operatorname{tp}(\vec{c} / \vec{b})$ such that $\overrightarrow{c^{\prime}} \downarrow_{\vec{b}} \vec{c}$ and $\vec{c} \downarrow_{\overrightarrow{c^{\prime}}} \vec{b}$. Then $\models r\left(\vec{b}, \overrightarrow{c^{\prime}}\right)$, $\vec{c} \notin \operatorname{acl}\left(\overrightarrow{c^{\prime}}\right)$ and $\overrightarrow{c^{\prime}} \notin \operatorname{acl}(\vec{c})$, and therefore $r(\vec{x}, \vec{c}) \wedge r\left(\vec{x}, \overrightarrow{c^{\prime}}\right)$ is finite. But this means that $\vec{b} \in \operatorname{acl}\left(\vec{c}, \overrightarrow{c^{\prime}}\right)$, a contradiction with $\vec{b} \notin \operatorname{acl}\left(\overrightarrow{c^{\prime}}\right)$ and $\vec{c} \downarrow_{\overrightarrow{c^{\prime}}} \vec{b}$.

$(2 \rightarrow 3)$ Trivial.

$(3 \rightarrow 1)$ Suppose $T$ is not weakly 1 -based. Adding constants to the language if necessary, by remark 2.8 we may assume that this is witnessed by $\operatorname{tp}(a b / c d)$ where $\operatorname{dim}(c d)=2$. So for any $a^{\prime} b^{\prime} \equiv_{c d} a b$ such that $a^{\prime} b^{\prime} \downarrow_{c d} a b$ we have $\operatorname{dim}\left(a b a^{\prime} b^{\prime}\right)=4$.

Let $r(x y, z t)=\operatorname{tp}(a b, c d)$.

We claim that if $a^{\prime} b^{\prime}$ realizes $\operatorname{tp}(a b)$ and $\operatorname{acl}\left(a^{\prime} b^{\prime}\right) \neq \operatorname{acl}(a b)$ then $r(a b, z t) \wedge r\left(a^{\prime} b^{\prime}, z t\right)$ has finitely many realizations. In other words, if $a^{\prime} b^{\prime} \equiv_{c d} a b$ and $\operatorname{acl}(a b) \neq \operatorname{acl}\left(a^{\prime} b^{\prime}\right)$, then $c, d \in \operatorname{acl}\left(a b a^{\prime} b^{\prime}\right)$.

Case 1: $a \downarrow_{c d} a^{\prime}$. Then $a^{\prime} b^{\prime} \downarrow_{c d} a b$, so $\operatorname{dim}\left(a b a^{\prime} b^{\prime}\right)=4$. Now

$$
\operatorname{dim}\left(a b a^{\prime} b^{\prime} c d\right)=\operatorname{dim}\left(a b a^{\prime} b^{\prime} / c d\right)+\operatorname{dim}(c d)=2+2=4=\operatorname{dim}\left(a b a^{\prime} b^{\prime}\right),
$$

hence $c, d \in \operatorname{acl}\left(a b a^{\prime} b^{\prime}\right)$.

Case 2: $\operatorname{acl}(a c d)=\operatorname{acl}\left(a^{\prime} c d\right)$. Since $\operatorname{acl}(a b) \neq \operatorname{acl}\left(a^{\prime} b^{\prime}\right), a^{\prime}$ or $b^{\prime}$ is not in $\operatorname{acl}(a b)$. Thus either $\operatorname{dim}\left(a b a^{\prime}\right)=3$ or $\operatorname{dim}\left(a b b^{\prime}\right)=3$. Either way, since $\operatorname{dim}\left(a b a^{\prime} b^{\prime} c d\right)=3$, we get $c, d \in \operatorname{acl}\left(a b a^{\prime} b^{\prime}\right)$.

Now, $r(x y, z t)$ is a complete almost quasidesign, as needed.

We can now summarize our results on equivalent definitions of weak local modularity / weak 1-basedness by putting them together with the results form [5]. 
Theorem 2.11. The following are equivalent for any geometric theory $T$

(1) $T$ is weakly locally modular;

(2) $T$ is weakly one-based;

(3) $T$ does not have a partial (complete) almost quasidesign;

(4) in any lovely pair $(M, P)$ of models of $T, \operatorname{acl}_{P}=\operatorname{acl}$;

(5) in any lovely pair $(M, P)$ of models of $T$, the small closure operator $\mathrm{scl}=$ $\operatorname{acl}(-\cup P)$ induces a modular pregeometry

From now on we will use the terms weakly 1-based and weakly locally modular interchangeably.

It is known (see e.g.[18]) that reducts of geometric theories are geometric. It is also known (see [20]) that 1-basedness is preserved by reducts in the cases of superstable theories of finite U-rank and stable groups (given that the group operation is intact). In the case of SU-rank 1 structures, the fact that 1-basedness is preserved by reducts follows from its characterization in [23]: reduct of a lovely pair is again lovely, and $T_{P}$ having SU-rank $\leq 2$ is also preserved. In the o-minimal group case, it is known (see [15]) that linear structures are exactly the reducts of ordered vector spaces over division rings, and thus linearity is preserved under reducts as well. Here we generalize these facts to the case of geometric theories.

Proposition 2.12. Weak 1-basedness is preserved by reducts.

Proof. Suppose $T^{-} \subset T$ is a reduct. We are working in a sufficiently saturated model of $T$. Its reduct is a sufficiently saturated model of $T^{-}$. If $T^{-}$is not weakly 1-based, it has a complete almost quasidesign $r(\vec{x}, \vec{y})$. Adding parameters to the language we may assume that $r$ is over $\emptyset$. We claim that $r$ is a partial almost quasidesign in the sense of $T$. Part (2) of the definition is clear since $\operatorname{acl}^{-}(A)$ is a subset of $\operatorname{acl}(A)$ for any set $A$. Suppose part (1) fails in $T$. Thus in $T r(\vec{x}, \vec{y})$ implies that $\vec{x} \in \operatorname{acl}(\vec{y})$ or $\vec{y} \in \operatorname{acl}(\vec{x})$. By compactness, $r(\vec{x}, \vec{y})$ implies (in $T$ ) a formula $\phi(\vec{x}, \vec{y}) \vee \psi(\vec{x}, \vec{y})$, where $\phi$ and $\psi$ witness $\vec{x} \in \operatorname{acl}(\vec{y})$ and $\vec{y} \in \operatorname{acl}(\vec{x})$ respectively.

Now, for any $\vec{b}$ and $\vec{c}$ such that $=r(\vec{b}, \vec{c}), \vec{b} \notin \operatorname{acl}^{-}(\vec{c})$ and $\vec{c} \notin \operatorname{acl}^{-}(\vec{b})$ (since $r$ is complete in $\left.T^{-}\right)$. Then for any $\vec{b} \vec{c} \models r(\vec{x}, \vec{y})$ we have either $\models \phi(\vec{b}, \vec{c})$ (i.e. $\vec{b} \in \operatorname{acl}(\vec{c})$ ) or $\models \psi(\vec{b}, \vec{c})$ (i.e. $\vec{c} \in \operatorname{acl}(\vec{b}))$, or both.

On the other hand, whenever $\models r(\vec{b}, \vec{c})$, there are infinitely many $\vec{c}^{\prime}$ and $\overrightarrow{b^{\prime}}$ such that $\models r\left(\vec{b}, \overrightarrow{c^{\prime}}\right)$ and $\models r\left(\overrightarrow{b^{\prime}}, \vec{c}\right)$. Now, for all but finitely many $\vec{c}^{\prime}$ we have $\models \phi\left(\vec{b}, \overrightarrow{c^{\prime}}\right)$ witnessing $\vec{b} \in \operatorname{acl}\left(\overrightarrow{c^{\prime}}\right)$ (since $\psi(\vec{b}, \vec{y})$ has finitely many solutions). Similarly, for all but finitely many $\overrightarrow{b^{\prime}}$ we have $\models \psi\left(\overrightarrow{b^{\prime}}, \vec{c}\right)$ witnessing $\vec{c} \in \operatorname{acl}\left(\overrightarrow{b^{\prime}}\right)$ (since $\psi(\vec{x}, \vec{c})$ has finitely many solutions).

Thus for any $n$ we can build a sequence $\vec{b}_{0} \vec{c}_{0} \vec{b}_{1} \vec{c}_{1} \vec{b}_{2} \vec{c}_{2} \ldots \vec{b}_{n} \vec{c}_{n}$ such that

$$
\begin{gathered}
\models r\left(\vec{b}_{i}, \vec{c}_{i}\right), \\
=r\left(\vec{b}_{i+1}, \vec{c}_{i}\right), \\
\vec{b}_{i} \in \operatorname{acl}\left(\vec{c}_{i}\right), \\
\vec{c}_{i} \in \operatorname{acl}\left(\vec{b}_{i+1}\right), \\
\vec{b}_{i+1} \notin \operatorname{acl}\left(\vec{c}_{i}\right), \\
\vec{c}_{i} \notin \operatorname{acl}\left(b_{i}\right) .
\end{gathered}
$$

Thus we have strict embeddings

$$
\operatorname{acl}\left(\vec{b}_{0}\right) \subset \operatorname{acl}\left(\vec{c}_{0}\right) \subset \operatorname{acl}\left(\vec{b}_{1}\right) \subset \operatorname{acl}\left(\vec{c}_{1}\right) \subset \ldots \subset \operatorname{acl}\left(\vec{b}_{n}\right) \subset \operatorname{acl}\left(\vec{c}_{n}\right) .
$$


Contradiction with finiteness of $\operatorname{dim}(\vec{c})$.

Our next goal is to compare weak local modularity with linearity. We start by recalling the definition from [18]:

Definition 2.13. Let $T$ be a geometric theory and let $M \models T$ be saturated. By a curve we mean a one dimensional subset of $M^{2}$. A family $\mathcal{F}$ of plane curves is said to be definable if it can be written as a family of fibers of a definable subset of $M^{2} \times M^{k}$, where the parameter set is the subset of $M^{k}$. A family $\mathcal{F}$ of plane curves is said to be interpretable if it can be written as a family of fibers of a definable subset of $M^{2} \times\left(M^{k} / E\right)$, where $E$ is a definable equivalence relation. We say $\mathcal{F}$ is normal if any two curves from $\mathcal{F}$ which are given by different parameters intersect at most finitely many times. We say that $T$ is linear if every interpretable normal family of plane curves has dimension $\leq 1$.

On has to be careful with the previous definition. In order for the dimension of an interpretable family of plane curves to be defined, we need to extend the notion of dimension from real tuples to imaginary tuples. In [13] Gagelman showed that the geometric theories $T$ where the notion of independence extends to the set of imaginary elements are those that are surgical. Recall that a geometric theory $T$ is surgical if whenever $X \subset M^{n}$ is definable and $\operatorname{dim}(X)=m$ then there is no definable equivalence relation $E$ on $X$ that has infinitely many classes of dimension $m$. The results from [13] together with the fact that thorn forking is the weakest notion of independence [12], show that $T$ is surgical if and only if $T$ is rosy of thorn rank one.

We will divide our discussion on normal families of plane curves into two cases. We will first deal with definable families in the setting of geometric theories. Then we will deal with the case of interpretable families when the underlying theory is rosy of thorn rank one.

For the following results we will use the tools of lovely pairs developed in [5]. In particular, we will use the fact that a theory $T$ is weakly locally modular if and only if the small closure in a saturated model of $T_{P}$ is modular.

Lemma 2.14. Let $T$ be a geometric theory and let $M$ be a saturated model of $T$. If $M$ has a definable normal family of plane curves of dimension $\geq 2$ then $T$ is not weakly locally modular.

Proof. We may assume that there is $N \preceq M$ such that $(M, N)$ is a lovely pair of models of $T$ and we write $P$ instead of $N$. For $A \subset M$ we write $\operatorname{scl}(A)$ for $\operatorname{acl}(A, P)$. Assume that $T$ is weakly locally modular so scl is modular. By hypothesis there is a 2-dimensional normal family of plane curves, say given by $\{C(x, y, \vec{a}, \vec{b}): \vec{a} \in \theta(\vec{z}, \vec{b})\}$ where $\theta(\vec{z}, \vec{b})$ defines a subset of $M^{k}$ and $\operatorname{dim}(\theta(\vec{z}, \vec{b}))=2$. We may assume that $\theta$ is defined over $\emptyset$.

We may assume that $\theta(\vec{a})=\theta\left(a_{1}, a_{2}, \vec{a}_{3}\right)$ and that whenever $\theta\left(a_{1}, a_{2}, \vec{a}_{3}\right)$ holds then $\vec{a}_{3} \in \operatorname{acl}\left(a_{1}, a_{2}\right)$. Let $\vec{a}=\left(a_{1}, a_{2}, \vec{a}_{3}\right) \in \theta$ be generic over $P$, let $c, d \in M$ be such that $C\left(c, d, a_{1}, a_{2}, \vec{a}_{3}\right)$ and choose $c$ independent from $a_{1} a_{2} P$. Let $X=$ $\operatorname{scl}(c, d), Y=\operatorname{scl}\left(a_{1}, a_{2}\right)$. Since scl is modular and $\operatorname{dim}(X \cup Y / P)=3$ we must have $\operatorname{dim}(X \cap Y / P)=1$. Let $t$ be real such that $\operatorname{scl}(t)=X \cap Y$. Note that $d \in \operatorname{scl}(c, t)$ and that $\operatorname{dim}\left(a_{1} a_{2} / t P\right)=1$. Let $\vec{p} \in P$ be such that $d \in \operatorname{acl}(c, t, \vec{p})$, $\operatorname{dim}\left(a_{1} a_{2} / t \vec{p}\right)=1$. Note that by genericity of $\left(a_{1}, a_{2}\right)$ we have $t \in \operatorname{acl}\left(a_{1}, a_{2}, \vec{p}\right)$. 
Let $\left(a_{1}^{\prime}, a_{2}^{\prime}, \vec{a}_{3}^{\prime}\right)=\operatorname{tp}\left(a_{1}, a_{2}, \vec{a}_{3} / t, c, d, \vec{p}\right)$ be independent from $c, t, a_{1}, a_{2}, \vec{p}$ over $c, t, \vec{p}$. Then whenever $c^{\prime}=\operatorname{tp}\left(c / a_{1}, a_{2}, \vec{a}_{3}, a_{1}^{\prime}, a_{2}^{\prime}, \vec{a}_{3}^{\prime}, \vec{p}\right)$ we have that

$$
\exists y\left(C\left(c^{\prime}, y, a_{1}, a_{2}, \vec{a}_{3}\right) \wedge C\left(c^{\prime}, y, a_{1}^{\prime}, a_{2}^{\prime}, \vec{a}_{3}^{\prime}\right)\right) .
$$

Since the type $\operatorname{tp}\left(c / a_{1}, a_{2}, \vec{a}_{3}, a_{1}^{\prime}, a_{2}^{\prime}, \vec{a}_{3}^{\prime}, \vec{p}\right)$ is not algebraic, the family of plane curves is not normal, a contradiction.

Lemma 2.15. Let $T$ be a thorn rank one rosy theory and let $M$ be a saturated model of $T$. If $M$ has a interpretable normal family of plane curves of dimension $\geq 2$ then $\mathrm{scl}$ is not modular.

Proof. As before we may assume that $(M, P)$ is a lovely pair of models of $T$. By hypothesis there is a 2-dimensional normal family of plane curves, say given by $\{C(x, y, \hat{a}): \hat{a} \in \theta\}$ where $\theta(\hat{z})$ defines a subset of $M^{e q}$ and $\operatorname{dim}(\theta(\hat{z}))=2$. We may assume that $\theta$ is defined over $\emptyset$. Let $a$ be a base for $\hat{a}$, so $\hat{a}=a_{E}$ for some definable equivalence relation $E$. We may write $a=\left(a_{1}, \ldots, a_{k}, \ldots, a_{n}\right)$, where $a_{1}, \ldots, a_{k}$ are independent and $a_{k+1}, \ldots, a_{n} \in \operatorname{acl}\left(a_{1}, \ldots, a_{k}\right)$. By the extension property, we may choose $a$ such that $\operatorname{dim}(a / P)=k$. Let $c, d \in M$ be such that $C(c, d, \hat{a})$ and choose $c$ independent from $a, P$. Let $X=\operatorname{scl}(c, d), Y=\operatorname{scl}(a)$. Since scl is modular and $\operatorname{dim}(X \cup Y / P)=1+\operatorname{dim}(Y / P)$ we must have $\operatorname{dim}(X \cap Y / P)=1$. Let $t$ be real such that $\operatorname{scl}(t)=X \cap Y$. Note that $d \in \operatorname{scl}(c, t)$ and that $\operatorname{dim}(a / t P)<\operatorname{dim}(a / P)$. Without loss of generality we may assume that $a_{k} \in \operatorname{acl}\left(a_{1}, \ldots, a_{k-1}, t, P\right)$. Let $\vec{p} \in P$ be such that $d \in \operatorname{acl}(c, t, \vec{p}), a_{k} \in \operatorname{acl}\left(a_{<k}, t, \vec{p}\right)$, by the exchange property we have $t \in \operatorname{acl}\left(a_{1}, \ldots, a_{k}, \vec{p}\right)$.

Let $b=\operatorname{tp}(a / \operatorname{acl}(t, c, d, \vec{p}))$ be independent from $c, t, a, \vec{p}$ over $c, t, \vec{p}$. Let $\hat{b}=$ $b_{E}$, so we get $\hat{a} \downarrow_{c t \vec{p}} \hat{b}$ and $c \notin \operatorname{acl}(a, b, t, \vec{p})$. Since $\operatorname{dim}(\hat{a} / c t \vec{p})=\operatorname{dim}(\hat{a} / c d \vec{p})=$ $\operatorname{dim}(\hat{a} / c d)=1$, we must have $\hat{a} \neq \hat{b}$. Then whenever $c^{\prime} \models \operatorname{tp}(c / a, b, \vec{p})$ we have that there is $y$ satisfying $C\left(c^{\prime}, y, \hat{a}\right)$ and $C\left(c^{\prime}, y, \hat{b}\right)$. Since the type $\operatorname{tp}(c / a, b, \vec{p})$ is not algebraic, the family of plane curves is not normal, a contradiction.

We will prove below a partial converse to the previous results using the proof of Proposition 2.10.

Definition 2.16. Let $T$ be a geometric theory and let $M$ be a saturated model of $T$. Let $\mathcal{F}=\{\psi(z, t, \vec{a}, \vec{b}): \vec{a}=\varphi(\vec{x}, \vec{b})\}$ be a family of plane curves. We say that $\mathcal{F}$ is generically normal if whenever $\vec{a}, \vec{a}^{\prime}=\varphi(\vec{x}, \vec{b})$ are such that $\operatorname{dim}\left(\vec{a} / \vec{a}^{\prime} \vec{b}\right) \geq 1$, we have that $\psi(z, t, \vec{a}, \vec{b}) \wedge \psi\left(z, t, \vec{a}^{\prime}, \vec{b}\right)$ is finite. We say that $T$ is generically linear if every generically normal family of plane curves has dimension $\leq 1$.

Proposition 2.17. Let $T$ be a geometric theory. If $T$ not weakly 1-based, then $T$ is not generically linear.

Proof. Let $M$ be a saturated model of $T$. Assume $T$ is not weakly 1-based, so this fact is witnessed by $\operatorname{tp}\left(a_{1} a_{2} / c d \vec{b}\right)$ where $\operatorname{dim}(c d / \vec{b})=2$. So for any $a_{1}^{\prime} a_{2}^{\prime} \equiv_{c d \vec{b}}$ $a_{1} a_{2}$ such that $a_{1}^{\prime} a_{2}^{\prime} \downarrow_{c d \vec{b}} a_{1} a_{2}$ we have $\operatorname{dim}\left(a_{1} a_{2} a_{1}^{\prime} a_{2}^{\prime}\right)=4$. Let $r\left(z t, x_{1} x_{2}\right) \stackrel{c a b}{=}$ $\operatorname{tp}\left(c d, a_{1} a_{2} / \vec{b}\right)$. As in the proof of Proposition 2.10, we have that if $a_{1}^{\prime} a_{2}^{\prime}$ realizes $\operatorname{tp}\left(a_{1} a_{2} / \vec{b}\right)$ and $\operatorname{acl}\left(a_{1}^{\prime} a_{2}^{\prime} \vec{b}\right) \neq \operatorname{acl}\left(a_{1} a_{2} \vec{b}\right)$ then $r\left(z t, a_{1} a_{2}\right) \wedge r\left(z t, a_{1}^{\prime} a_{2}^{\prime}\right)$ has finitely many realizations. By compactness there is a uniform bound $m$ for these realizations. Choose a formula $\psi\left(z, t, x_{1}, x_{2}, \vec{b}\right) \in r\left(z, t, x_{1}, x_{2}\right)$ such that $\psi\left(z, t, a_{1}, a_{2}, \vec{b}\right)$ is one dimensional and such that whenever $a_{1}^{\prime} a_{2}^{\prime}$ realizes $\operatorname{tp}\left(a_{1} a_{2} / \vec{b}\right)$ and $\operatorname{acl}\left(a_{1}^{\prime} a_{2}^{\prime} \vec{b}\right) \neq$ 
$\operatorname{acl}\left(a_{1} a_{2} \vec{b}\right)$ then $\psi\left(z, t, a_{1} a_{2}, \vec{b}\right) \wedge \psi\left(z, t, a_{1}^{\prime} a_{2}^{\prime}, \vec{b}\right)$ has at most $m$ realizations. By compactness, there is a formula $\varphi\left(x_{1}, x_{2}, \vec{b}\right) \in \operatorname{tp}\left(a_{1}, a_{2} / \vec{b}\right)$, such that if $a_{1}^{\prime} a_{2}^{\prime}$ realizes $\varphi\left(x_{1}, x_{2}, \vec{b}\right)$ then $\psi\left(z, t, a_{1}^{\prime}, a_{2}^{\prime}, \vec{b}\right)$ is one dimensional (in the variables $z, t$ ). Making $\varphi\left(x_{1}, x_{2}, \vec{b}\right)$ and $\psi\left(z, t, x_{1}, x_{2}, \vec{b}\right)$ smaller if necessary, whenever $a_{1} a_{2}, a_{1}^{\prime} a_{2}^{\prime}$ are realizations of $\varphi\left(x_{1}, x_{2}, \vec{b}\right)$ such that $\operatorname{acl}\left(a_{1} a_{2} \vec{b}\right) \neq \operatorname{acl}\left(a_{1}^{\prime} a_{2}^{\prime} \vec{b}\right)$ we have that $\psi\left(z, t, a_{1}, a_{2}, \vec{b}\right) \wedge \psi\left(z, t, a_{1}^{\prime}, a_{2}^{\prime}, \vec{b}\right)$ has at most $m$ realizations. Thus, generically $\left\{\psi\left(z, t, a_{1}, a_{2}, \vec{b}\right):\left(a_{1}, a_{2}\right) \models \varphi\left(x_{1}, x_{2}, \vec{b}\right)\right\}$ is a 2-dimensional family of plane curves and $T$ is not generically linear.

\section{3. $\omega$-CATEGORICAL CASE}

One of the main consequences of one-basedness in (non-trivial) stable, and to some extent, simple geometric theories was definability or type-definability of infinite groups in $T^{e q}$. In the o-minimal case, groups appear naturally in the linear case, as a consequence of the Trichotomy theorem. It is well-known that the geometry of a non-trivial locally modular (one-based) strongly minimal structure is projective or affine over a division ring, and the corresponding vector space is actually definable. This is no longer the case for a non-trivial 1-based SU-rank 1 theory, but De Piro and Kim [9] show, using canonical bases, that an $\omega$-categorical non-trivial 1-based SU-rank 1 theory interprets an infinite vector space over a finite field. Thus our best hope at this point is to obtain a group in the case of an $\omega$-categorical non-trivial weakly 1-based geometric theory. In the case of geometric theories, since canonical bases are not readily available, we use the lovely pairs approach developed in [23].

First we note that the weak 1-basedness assumption implies the preservation of $\omega$-categoricity when passing to the theory of lovely pairs.

The following is a generalization of Proposition 5.15 from [23], and its proof also improves the estimate on the size of a $P$-independent extension from Lemma 5.14. of [23].

Proposition 3.1. Let $T$ be an $\omega$-categorical weakly 1-based geometric theory. Then $T_{P}$ is $\omega$-categorical.

Proof. Let $\vec{a} \vec{b}$ be a tuple of length $n$ in a lovely pair, such that $\vec{a} \in \operatorname{acl}(\vec{b} P)$ and $\vec{b}$ is independent over $P$. Let $\vec{p} \in P$ be such that $\vec{a} \in \operatorname{acl}(\vec{b} \vec{p})$. By weak 1-basedness, there is $\vec{a}^{\prime} \vec{b}^{\prime} \models t p(\vec{a} \vec{b} / \bar{p})$ such that $\vec{a} \vec{b} \downarrow_{\vec{p}} \vec{a}^{\prime} \vec{b}^{\prime}$ and $\vec{a} \vec{b} \downarrow_{\vec{a}^{\prime} \vec{b}^{\prime}} \vec{p}$. Then $\overrightarrow{b^{\prime}}$ is independent over $\bar{p} \vec{a} \vec{b}$, so we may assume that $\vec{b}^{\prime} \in P$. It follows that $\vec{a}^{\prime} \in P \vec{a} \vec{b} \downarrow_{\vec{a}^{\prime} \vec{b}^{\prime}} P$. Thus any $n$-tuple can be extended to a $P$-independent set of size $2 n$ (in fact, by its own $\mathcal{L}$-conjugate). Then by uniform local finiteness of acl in $T$, there is a function $f: \omega \rightarrow \omega$ such that any $n$ tuple embeds in a $P$-independent algebraically closed set of size $f(n)$. Since for such sets $L_{P}$-type is determined by quantifier free $L_{P}$-type, we have finitely many $n$-types in $T_{P}$ for any $n$. Thus $T_{P}$ is $\omega$-categorical.

As in [23], if $T$ is a non-trivial weakly locally modular geometric theory, then the geometry of the small closure (the quotient geometry, or the associated geometry of $(M$, acl $(-\cup P(M))))$ is split into a disjoint union of infinite-dimensional projective geometries over division rings (and possibly a trivial geometry) by the equivalence relation " $x=y$ or $|c l(x, y)| \geq 3$ ".

If $T$ is weakly 1 -based and $\omega$-categorical, then by the above proposition, $T_{P}$ is also $\omega$-categorical and the relations $y \in \operatorname{acl}\left(y_{1}, \ldots, y_{n}, P\right)$ and the equivalence 
relation $\operatorname{acl}(x, P)=\operatorname{acl}(y, P)$ are $L_{P}$-definable. Thus the geometry of the small closure is interpretable in $T_{P}$ and the relations $x \in \operatorname{cl}\left(y_{1}, \ldots, y_{n}\right)$ on its elements are definable in $\left(T_{P}\right)^{e q}$. Clearly, the equivalence relation " $x=y$ or $|c l(x, y)| \geq 3$ " is also definable, and thus each of the projective geometries over division rings mentioned above, viewed as a structure where the only relations are given by $x \in \operatorname{cl}\left(y_{1}, \ldots, y_{n}\right)$, $n \geq 1$, is definable in $\left(T_{P}\right)^{e q}$ (as a quotient of the home sort). Note that each of these geometries is an $\omega$-categorical structure, and in the superrosy thorn-rank 1 case, by Fact 2.2 it is superrosy of thorn-rank at most 2 .

Let $(V,+, \lambda \cdot)_{\lambda \in F}$ be an infinite dimensional vector space over a division $\operatorname{ring} F$. By $\operatorname{Geom}(V)$ we denote the associated geometry of $(V, S p a n)$ viewed as a structure $\left(G, x \in \operatorname{cl}\left(y_{1}, \ldots, y_{n}\right)\right)_{n \geq 1}$. Note that $a \in \operatorname{cl}(\bar{b})$ does not imply $a \in \operatorname{acl}(\bar{b})$ in this language unless $F$ is finite.

Our goal is to show that the division rings above are actually finite fields. Then $\operatorname{Geom}(V)$ is a non-trivial $\omega$-categorical strongly minimal structure, and it is wellknown that such a theory interprets an infinite group (namely, a vector space over a finite field).

The following proposition shows that for an infinite $F$, Th $(\operatorname{Geom}(V))$ has a thorn-forking chains of any finite length. It follows that if $T$ is a weakly 1-based superrosy theory of thorn-rank 1 , then all the division rings above are finite (since, as noted above, hence $\operatorname{Geom}(V)$, will have thorn-rank at most 2 ), and thus $T_{P}$ interprets an infinite group (a vector space over a finite field).

When working in $\operatorname{Geom}(V)$, for any $v \in V$, by $v^{*}$ we denote $\operatorname{Span}(v)$ as an element of $\operatorname{Geom}(V)$.

Proposition 3.2. Suppose $V$ is a vector space over an infinite division ring. Let $v_{1}, \ldots, v_{n} \in V$ be linearly independent. Let $u_{k}=v_{1}+v_{2}+\ldots+v_{k}$. Then for any $1<$ $k \leq n, \operatorname{tp}\left(\left(u_{n}^{*} / v_{1}^{*}, \ldots, v_{n}^{*}, u_{2}^{*}, \ldots, u_{k}^{*}\right)\right.$ thorn-divides over $\left\{v_{1}^{*}, \ldots, v_{n}^{*}, u_{2}^{*}, \ldots, u_{k-1}^{*}\right\}$.

Proof. Note that $u_{n}^{*}$ satisfies the formula

$$
\phi\left(x, u_{k}^{*}, v_{k+1}^{*}, \ldots, v_{n}^{*}\right)=x \in \operatorname{cl}\left(u_{k}^{*}, v_{k+1}^{*}, \ldots, v_{n}^{*}\right) \wedge x \notin c l\left(v_{k+1}^{*}, \ldots, v_{n}^{*}\right) .
$$

We will show that $\phi(x)$ strongly divides over $\left\{v_{1}^{*}, \ldots, v_{n}^{*}, u_{2}^{*}, \ldots, u_{k-1}^{*}\right\}$. We are now working in a saturated elementary extension $G$ of $\operatorname{Geom}(V)$.

CLAIM: $\left\{\phi\left(x, a, b_{1}, \ldots, b_{n-k}\right) \mid a \vec{b} \models t p\left(u_{k}^{*}, v_{k+1}^{*}, \ldots, v_{n}^{*} / v_{1}^{*}, \ldots, v_{n}^{*}, u_{2}^{*}, \ldots, u_{k-1}^{*}\right)\right\}$ is 2-inconsistent.

Proof of the Claim: Note that for any $a \vec{b}$ as above, $b_{1}=v_{k+1}^{*}, \ldots, b_{n-k}=v_{n}^{*}$ and $a$ satisfies $\psi\left(y, u_{k-1}^{*}, v_{k}^{*}\right)=y \in c l\left(u_{k-1}^{*}, v_{k}^{*}\right)$ (since this holds for $\left.u_{k}^{*}\right)$. Now, if $a, a^{\prime} \in G$ are two distinct realizations of $\psi\left(y, u_{k-1}^{*}, v_{k}^{*}\right)$, then

$$
\phi\left(x, a, v_{k+1}^{*}, \ldots, v_{n}^{*}\right) \wedge \phi\left(x, a^{\prime}, v_{k+1}^{*}, \ldots, v_{n}^{*}\right)
$$

is inconsistent. Indeed, we may assume that $a, a^{\prime} \in \operatorname{Geom}(V)$, so $a=w_{1}^{*}$ and $a^{\prime}=w_{2}^{*}$ for some linearly independent $w_{1}, w_{2} \in V$. Now, if $\phi\left(x, w_{1}^{*}, v_{k+1}^{*}, \ldots, v_{n}^{*}\right) \wedge$ $\phi\left(x, w_{2}^{*}, v_{k+1}^{*}, \ldots, v_{n}^{*}\right)$ is realized by some $p^{*}$ (where $p \in V$ ), then from the definition of $\phi$,

$$
p=\gamma_{1} w_{1}+\mu_{1} v_{k+1}+\ldots+\mu_{n-k} v_{n}=\gamma_{2} w_{2}+\xi_{1} v_{k+1}+\ldots+\xi_{n-1} v_{n},
$$

where $\gamma_{1}, \gamma_{2} \neq 0$. Thus, $\gamma_{1} w_{1}-\gamma_{2} w_{2} \in \operatorname{Span}\left(v_{k+1}, \ldots, v_{n}\right)$. On the other hand, $\gamma_{1} w_{1}-\gamma_{2} w_{2} \neq 0$ (by linear independence of $w_{1}$ and $w_{2}$ ) and $\gamma_{1} w_{1}-\gamma_{2} w_{2} \in$ $\operatorname{Span}\left(v_{1}, \ldots, v_{k-1}, v_{k}\right)$ since $w_{1}^{*}, w_{2}^{*} \in \operatorname{cl}\left(u_{k-1}^{*}, v_{k}^{*}\right)$. Thus $\operatorname{Span}\left(v_{1}, \ldots, v_{k-1}, v_{k}\right)$ and $\operatorname{Span}\left(v_{k+1}, \ldots, v_{n}\right)$ have a non-zero vector in their intersection, a contradiction 
with the linear independence of $v_{1}, \ldots, v_{n}$. This proves the Claim, and hence $\phi(x)$ strongly divides over $\left\{v_{1}^{*}, \ldots, v_{n}^{*}, u_{2}^{*}, \ldots, u_{k-1}^{*}\right\}$, as needed.

Corollary 3.3. If $T$ is a $\omega$-categorical weakly 1-based thorn rank one theory, then $T_{P}$ interprets an infinite group.

The assumption of $T$ being superrosy of thorn rank one seems quite artificial, and we therefore conjecture that the above result holds for any $\omega$-categorical weakly one-based geometric theory. A key issue here is to understand the theory $T h(\operatorname{Geom}(V))$ when $V$ is infinite-dimensional over an infinite division ring. So far we know that $T h(G e o m(V))$ has infinite thorn-forking (even thorn-dividing) chains, and any model of $T h($ Geom $(V))$ is an infinite-dimensional projective geometry over an infinite (and possibly different) division ring. However the following questions remain open.

Question 3.4. Let $V$ be an infinite-dimensional vector space over an infinite division ring, and let $T=T h(\operatorname{Geom}(V))$.

(1) Is $T \omega$-categorical?

(2) Is T stable?

(3) Does $T$ have trivial algebraic closure?

(4) Does T have quantifier elimination?

(5) What happens when we vary the (infinite) division ring?

\section{Independence in $T_{P}$ FOR $T$ Weakly 1 -Based}

We know from Fact 1.3, that for $T$ a rosy theory of thorn rank one, the associated theory $T_{P}$ of lovely pairs of models of $T$ is again rosy of thorn rank $\leq \omega$. It is an interesting question which other properties of $T$ are preserved in $T$. We start by generalizing the notion of weak 1-based theories to the setting of rosy theories.

Definition 4.1. Let $T$ be a rosy theory. We say that $T$ is weakly 1 -based if whenever $M \models T$ is saturated, $B \subset M$ and $\vec{a} \in M$ there is a superset $C$ of $B$ independent from $\vec{a}$ over $B$ such that whenever $\vec{a}^{\prime} \models \operatorname{tp}(\vec{a} / C)$ is independent from $\vec{a}$ over $C$, we have $\vec{a} \downarrow_{\vec{a}^{\prime}} B$.

Note that for a simple $T$, a canonical base argument shows that weak 1-basedness coincides with 1-basedness. The goal of this section is to show that whenever $T$ is weakly 1-based rosy rank one theory then $T_{P}$ is again weakly 1-based. We only succeeded in doing this under some extra assumptions.

Lemma 4.2. Let $T$ be a weakly 1-based rosy theory. Let $M \models T$ be sufficiently saturated, let $\vec{a} \in M, B \subset M$ and let $C \supset B$ be such that $\vec{a} \downarrow_{B} C$ and whenever $\vec{a}^{\prime} \models \operatorname{tp}(\vec{a} / C)$ is independent from $\vec{a}$ over $C$, we have $\vec{a} \downarrow_{\vec{a}^{\prime}} B$. Let $D \models \operatorname{tp}(C / B \vec{a})$, then whenever $\vec{a}^{\prime} \models \operatorname{tp}(\vec{a} / D)$ is independent from $\vec{a}$ over $D$, we have $\vec{a} \downarrow_{\vec{a}^{\prime}} B$.

Proof. Clear.

Remark 4.3. Let $M \models T$ be sufficiently saturated, let $\vec{a} \in M, B \subset M$ and assume that there is a set $C \supset B$ with $\vec{a} \downarrow_{B} C$ such that whenever $\vec{a}^{\prime} \models \operatorname{tp}(\vec{a} / C)$ is independent from $\vec{a}$ over $C$, we have $\vec{a} \downarrow_{\vec{a}^{\prime}} B$. Also assume that $\vec{b} \in M$ and that there is a set $D \supset B$ with $\vec{a} \vec{b} \downarrow_{B} D$ such that whenever $\vec{a}^{\prime} \vec{b}^{\prime}=\operatorname{tp}(\vec{a} \vec{b} / D)$ is independent from $\vec{a} \vec{b}$ over $D$, we have $\vec{a} \vec{b} \downarrow_{\vec{a}^{\prime} \vec{b}^{\prime}} B$. Let $C^{\prime} \equiv_{B \vec{a}} C$ be such that 
$C^{\prime} \downarrow_{B \vec{a}} D \vec{b}$ and let $E=D \cup C^{\prime}$. Then whenever $\vec{a}^{\prime} \vec{b}^{\prime} \models \operatorname{tp}(\vec{a} \vec{b} / E)$ is independent from $\vec{a} \vec{b}$ over $E$, we have $\vec{a} \vec{b} \downarrow_{\vec{a}^{\prime} \vec{b}^{\prime}} B$ and $\vec{a} \downarrow_{\vec{a}^{\prime}} B$.

Proof. Let $C^{\prime} \equiv_{B \vec{a}} C$ be such that $C^{\prime} \downarrow_{B \vec{a}} D \vec{b}$ and let $E=D \cup C^{\prime}$. Note that $C^{\prime} \downarrow_{B} \vec{a}$. By transitivity we get $C^{\prime} \downarrow_{B} D \vec{a} \vec{b}$ and $C^{\prime} \downarrow_{B D} \vec{a} \vec{b}$. Applying symmetry and transitivity we get $\vec{a} \vec{b} \downarrow_{B} E$. Let $\vec{a}^{\prime} \vec{b}^{\prime} \models \operatorname{tp}(\vec{a} \vec{b} / E)$ be such that $\vec{a}^{\prime} \vec{b}^{\prime} \downarrow_{E} \vec{a} \vec{b}$. In particular, since $\vec{a} \vec{b} \downarrow_{D} E$, we have $\vec{a}^{\prime} \vec{b}^{\prime}=\operatorname{tp}(\vec{a} \vec{b} / D)$ and $\vec{a}^{\prime} \vec{b}^{\prime} \downarrow_{D} \vec{a} \vec{b}$. Thus $\vec{a} \vec{b} \downarrow_{\vec{a}^{\prime} \vec{b}^{\prime}} B$. We also have $\vec{a}^{\prime} \downarrow_{E} \vec{a}$ and $\vec{a} \downarrow_{C^{\prime}} E$, so $\vec{a}^{\prime} \downarrow_{C^{\prime}} \vec{a}$. By Lemma 4.2 $\vec{a} \downarrow_{\vec{a}^{\prime}} B$.

Notation 4.4. Let $(M, P) \models T_{P}$ be a saturated model. We use the word independence for acl-independence and we write $\downarrow$ for the acl-independence relation. We use the word $T_{P}$-independent for $p$-independence in models of $T_{P}$ and we write the corresponding independence relation as $\downarrow^{P}$.

We will need the following result from the proof of [5, Proposition 4.8]

Fact 4.5. Let $T$ be a weakly locally modular thorn rank one theory and let $(M, P) \models$ $T_{P}$. Let $a \in M, A \subset B \subset M$ and assume that $a \in \operatorname{acl}(A P) \backslash \operatorname{acl}(A)$ and that $a \in \operatorname{acl}(B P) \backslash \operatorname{acl}(B)$. Then $\operatorname{tp}_{P}(a / B)$ does not $p$-fork over $A$.

Notation 4.6. Let $a_{1}, \ldots, a_{n} \in M$. We write $a_{<1}$ for $\emptyset$ and for $1<i \leq n+1$, we write $a_{<i}$ for $\left(a_{1}, \ldots, a_{i-1}\right)$.

We will also assume the following condition:

Assumption 4.7. Let $T$ be a weakly 1-based geometric thorn rank one theory and let $(M, P) \models T_{P}$. Let $A \subset B \subset M$ and let $\vec{a}=\left(a_{1}, \ldots, a_{n}, a_{n+1}, \ldots, a_{m}, a_{m+1}, \ldots, a_{l}\right) \in$ $M$, where $\left(a_{1}, \ldots, a_{n}\right)$ is a $P \cup A$-independent tuple, for $i=n+1, \ldots, m a_{i} \in$ $\operatorname{acl}\left(a_{<i} P A\right) \backslash \operatorname{acl}\left(a_{<i} A\right)$ and for $i=m+1, \ldots, l \quad a_{i} \in \operatorname{acl}\left(a_{<i} A\right)$.

Then $\operatorname{tp}_{P}(\vec{a} / B)$ does not thorn fork over $A$ if and only if $\left(a_{1}, \ldots, a_{n}\right)$ is a $P \cup B$ independent tuple and for $i=n+1, \ldots, m a_{i} \in \operatorname{acl}\left(a_{<i}, P, B\right) \backslash \operatorname{acl}\left(a_{<i}, B\right)$.

In the above assumption, we know that the right property always implies the left property, we assume left to right.

Proposition 4.8. Assume that $T$ is a weakly 1-based geometric thorn rank one theory satisfying assumption 4.7. Then $T_{P}$ is also weakly 1-based.

Proof. Let $(M, P) \models T_{P}$ be saturated, let $\vec{a} \in M$ be a finite tuple and let $A \subset M$ be a set. We will write $\vec{a}=\left(a_{1}, \ldots, a_{n}, a_{n+1}, \ldots, a_{m}, a_{m+1}, \ldots, a_{l}\right)$ where $\left(a_{1}, \ldots, a_{n}\right)$ is a $P \cup A$-independent tuple, for $i=n+1, \ldots, m a_{i} \in \operatorname{acl}\left(a_{<i} P A\right) \backslash \operatorname{acl}\left(a_{<i} A\right)$ and for $i=m+1, \ldots, l a_{i} \in \operatorname{acl}\left(a_{<i} A\right)$. We need to find a superset $E \supset A$ such that $\vec{a} \downarrow_{A}^{P} E$ and whenever $\vec{a}^{\prime} \models \operatorname{tp}_{P}(\vec{a} / E)$ is such that $\vec{a} \downarrow_{E}^{P} \vec{a}^{\prime}$ then $\vec{a} \downarrow_{\vec{a}^{\prime}}^{P} A$.

Let $\vec{p}=\left(p_{1}, \ldots, p_{t}\right) \in P$ be an independent tuple over $A$ such that $a_{n+1}, \ldots, a_{m} \in$ $\operatorname{acl}\left(a_{1}, \ldots, a_{n}, p_{1}, \ldots, p_{t}, A\right)$. By hypothesis, there is a set $D \supset A$ such that $\vec{a} \vec{p} \downarrow_{A} D$ and whenever $\vec{a}^{\prime} \vec{p}^{\prime} \models \operatorname{tp}(\vec{a} \vec{p} / D)$ is such that $\vec{a} \vec{p} \downarrow_{D} \vec{a}^{\prime} \vec{p}^{\prime}$, then $\vec{a} \vec{p} \downarrow_{\vec{a}^{\prime} \vec{p}^{\prime}} A$. Again by hypothesis, there is a set $C \supset A$ such that $\vec{a} \downarrow_{A} C$ and whenever $\vec{a}^{\prime} \models \operatorname{tp}(\vec{a} / C)$ is such that $\vec{a} \downarrow_{C} \vec{a}^{\prime}$, then $\vec{a} \downarrow_{\vec{a}^{\prime}} A$. By the previous remark, we can find $E$ such that $E \downarrow_{A} \vec{a} \vec{p}$ and whenever $\vec{a}^{\prime} \vec{p}^{\prime} \models \operatorname{tp}(\vec{a} \vec{p} / E)$ is independent from $\vec{a} \vec{p}$ over $E$, we have $\vec{a} \vec{p} \downarrow_{\vec{a}^{\prime} \vec{p}^{\prime}} A$ and $\vec{a} \downarrow_{\vec{a}^{\prime}} A$. 
By Lemma 4.2 we may choose $E$ such that $E \downarrow_{A \vec{a} \vec{p}} P$.

Claim $\vec{a} \downarrow_{A}^{P} E$

From the previous conditions, we have $\vec{a} \vec{p} \downarrow_{A} E$ and $E \downarrow_{A} \vec{a} \vec{p} P$. Thus $a_{1}, \ldots, a_{n}$ is a $P \cup E$-independent tuple, $a_{i} \in \operatorname{acl}\left(a_{<i}, p_{1}, \ldots, p_{t}, E\right) \backslash \operatorname{acl}\left(a_{<i}, E\right)$ for $i=n+$ $1, \ldots, m$. Since $T$ is weakly locally modular, the claim follows from Fact 4.5.

Now let $\vec{a}^{\prime} \models \operatorname{tp}_{P}(\vec{a} / E)$ be such that $\vec{a}^{\prime} \downarrow_{E}^{P} \vec{a}$.

Claim $\vec{a} \underset{\vec{a}^{\prime}}{P} A$

By Assumption $4.7 a_{1}, \ldots, a_{n}$ is an $E \vec{a}^{\prime} \cup P$-independent $n$-tuple, so it is also a $\vec{a}^{\prime} \cup P$-independent $n$-tuple. Since $\vec{a}^{\prime} \downarrow_{E}^{P} \vec{a}$ we have $\vec{a}^{\prime} \downarrow_{E} \vec{a}$ and thus by $(0) \vec{a} \downarrow_{\vec{a}^{\prime}} A$. In particular, this shows that $a_{m+1}, \ldots, a_{l} \in \operatorname{acl}\left(a_{1}, \ldots, a_{m}, \vec{a}^{\prime}\right)$. It remains to show that $a_{n+1}, \ldots, a_{m} \in \operatorname{acl}\left(a_{1}, \ldots, a_{n}, \vec{a}^{\prime}, P\right)$.

Let $\vec{q}=\left(q_{1}, \ldots, q_{t}\right) \models \operatorname{tp}_{P}\left(p_{1}, \ldots, p_{t} / E \vec{a}\right)$ be such that $\vec{q} \downarrow_{\vec{a} E}^{P} \vec{a}^{\prime}$. By transitivity, we get $\vec{a} \vec{q} \downarrow_{E}^{P} \vec{a}^{\prime}$. Now let $\vec{q}^{\prime} \in P$ be such that $\operatorname{tp}_{P}(\vec{a} \vec{q} / E)=\operatorname{tp}_{P}\left(\vec{a}^{\prime} \vec{q}^{\prime} / E\right)$, we may choose $\vec{q}^{\prime}$ such that $\vec{q}^{\prime} \downarrow_{E \vec{a}^{\prime}}^{P} \vec{a} \vec{q}$ and by symmetry and transitivity we get $\vec{a} \vec{q} \downarrow_{E}^{P} \vec{a}^{\prime} \vec{q}^{\prime}$. From this we conclude $\vec{a} \vec{q} \downarrow_{E} \vec{a}^{\prime} \vec{q}^{\prime}$ and by (0) $\vec{a} \vec{q} \downarrow_{\vec{a}^{\prime} \vec{q}^{\prime}} A$. Since $a_{n+1}, \ldots, a_{m} \in$ $\operatorname{acl}\left(a_{1}, \ldots, a_{n}, \vec{q}, A\right)$, we get $a_{m+1}, \ldots, a_{l} \in \operatorname{acl}\left(a_{1}, \ldots, a_{n}, \vec{a}^{\prime}, \vec{q}, \vec{q}^{\prime}\right)$ as desired.

Corollary 4.9. Let $T$ be the theory of an o-minimal ordered vector space and let $T_{P}$ be the corresponding theory of lovely pairs. Then $T_{P}$ is weakly 1-based.

Proof. Since the algebraic closure coincides with the linear span, assumption 4.7 holds and thus by Proposition 4.8 the result follows.

Corollary 4.10. Let $T$ be an $S U$-rank one theory and let $T_{P}$ be the corresponding theory of lovely pairs. Then $T_{P}$ is weakly 1-based.

Proof. Since $T$ is simple of $S U$-rank one, $T$ is 1-based, $T_{P}$ is supersimple and forking and thorn forking coincide in models of $T_{P}$. By [23, Corollary 3.9] assumption 4.7 holds and thus by Proposition 4.8 the result follows.

Note that the previous result is known in a more general context. It is proved in [3] that if $T$ is simple 1-based and the theory $T_{P}$ of lovely pairs is first order, then $T_{P}$ is again 1-based.

We know from section 2 that in the geometric case, weak 1-basedness is preserved by reducts. As we mentioned earlier, it is known that reducts of 1-based superstable theories of finite U-rank are 1-based.

Question 4.11. Is a reduct of a weakly 1-based superrosy theory of finite thorn rank again weakly 1-based?

By Fact 2.2, for a weakly 1-based superrosy p-rank 1 geometric theory $T, T_{P}$ is superrosy of $\mathrm{p}$-rank $\leq 2$. We also know from [15], that linear o-minimal structures with global addition are precisely the reducts of ordered vector spaces. Since the reducts of lovely pairs are again lovely, a positive answer to the above question, together with Corollary 4.9, would imply preservation of weak 1-basedness (linearity) when passing to $T_{P}$ in the additive o-minimal case. 


\section{EXAmples}

5.1. Adding a generic predicate. In this section we assume the reader is familiar with the work of Chatzidakis and Pillay in random predicates [8]. We will show that if a theory is geometric and weakly 1-based then any of its completions with a random predicate is again weakly 1-based.

Fix $T$ a complete theory in a language $\mathcal{L}$. We will assume that $\mathcal{L}$ contains a unary predicate symbol $S$ (which could be equality) and we let $\mathcal{L}^{R}$ be the language $\mathcal{L}$ augmented with a new unary predicate symbol $R$ (we use the letter $R$ instead of the usual notation $P$, since we use $P$ in earlier parts of the paper to denote a predicate in a lovely pair). It is proved in [8] that the theory $T \cup\{\forall x R(x) \Longrightarrow S(x)\}$ has a model companion $T^{R, S}$. The theory $T^{R, S}$ may not be complete.

Our results rely heavily on the following facts:

Fact 5.1. [8, Corollary 2.6,(3)] The algebraic closure in models of $T^{R, S}$ coincides with the algebraic closure in the sense of $T$.

For models of $T^{R, S}$ we will write acl for the algebraic closure.

Fact 5.2. [8, Remark 2.12,(4)] If T eliminates $\exists^{\infty}$ then $T^{R, S}$ also eliminates $\exists^{\infty}$.

First observe that since $T$ is geometric, acl has the exchange property in models of $T^{R, S}$ and thus $T^{R, S}$ is pregeometric. Also, by the previous fact, $T^{R, S}$ eliminates $\exists^{\infty}$, so in fact $T^{R, S}$ is a geometric theory.

Lemma 5.3. Assume that $T$ is a geometric theory which is weakly 1-based. Then any completion of $T_{R, S}$ is weakly 1-based.

Proof. Let $M \models T^{R, S}$ be saturated, let $\vec{a} \in M$ and let $B \subset M$ be a set. By hypothesis there is a superset $C$ of $B$ with $\vec{a} \downarrow_{B} C$ such that whenever $\vec{a}^{\prime} \models \operatorname{tp}(\vec{a} / C)$ is acl-independent from $\vec{a}$ over $C$, we have $\vec{a} \mathfrak{\downarrow}_{\vec{a}^{\prime}} B$. Since algebraic independence in the sense of $T$ and $T^{R, S}$ coincide, $C$ is a witness for the desired property in $T^{R, S}$.

5.2. The structure induced on the predicate of a lovely pair. In this section we study the structure induced on the predicate of the lovely pair by the large model. Our presentation follows closely the one from Pillay and Vassiliev [22]. Let $T$ be a geometric theory in a language $\mathcal{L}$ with quantifier elimination and let $(M, P)$ be a lovely pair of models of $T$. For each $\mathcal{L}$-formula $\varphi(x)$ with parameters in $M$, we introduce a new predicate symbol $R_{\varphi}(x)$. Let $\mathcal{L}^{*}$ be the resulting language. We denote by $M^{*}$ the structure $M$ with the natural interpretation for the new relations and $P^{*}$ the substructure with universe $P$. Finally $T^{*}$ stands for the theory of $P^{*}$. Note that the language $\mathcal{L}^{*}$ and the theory $T^{*}$ depend on the choice of $M$. We denote the algebraic closure in models if $T$ by acl and in models of $T^{*}$ by acl*.

We will characterize acl* in terms of acl and $M$, prove that $T^{*}$ is also a geometric theory and that if $T$ is weakly 1 -based then $T^{*}$ is again weakly 1 -based.

Following [2, 22], we say that $(M, P)$ eliminates the quantifier $\exists y \in P$ if for every formula $\varphi(\vec{x}, \vec{y}, \vec{z})$ and $\vec{a} \in M$ there exists a formula $\psi(\vec{x}, \vec{w})$ and $\vec{b} \in M$ such that for all $\vec{c} \in P$,

$M \models \psi(\vec{c}, \vec{b})$ if and only if $(M, P) \models \exists d \in P \varphi(\vec{c}, d, \vec{a})$

It is clear that $(M, P)$ eliminates the quantifier $\exists y \in P$ if and only if $T^{*}$ has quantifier elimination. 
Lemma 5.4. The theory $T^{*}$ eliminates quantifiers.

Proof. Note that $T^{*}$ is a reduct of $T h_{M}(M, P)$, the theory of the pair with all the elements of $M$ added as constants. Take a saturated extension $(N, P)$ of $T h_{M}(M, P)$. Then the reduct of $P(N)$ to the language of $T^{*}$ is a saturated model of $T^{*}$.

Now, $(N, P)$ is still a lovely pair, and $M$ is $P$-independent in $(N, P)$. So any two tuples in $P(N)$ realizing the same quantifier free types in $T^{*}$, and hence the same $L$-type over $M$, actually realize the same $L_{P}$-type over $M$, and hence the same $T^{*}$ type. By saturation of $P(N)$ as a model of $T^{*}, T^{*}$ has quantifier elimination.

Lemma 5.5. Assume that $T$ is a geometric theory. Then $T^{*}$ is also geometric. Furthermore acl ${ }^{*}$ coincides with $\operatorname{acl}_{M}$ restricted to $P$.

Proof. Let $(N, P)$ be a saturated model of $T h_{M}(M, P)$. Let $B \subset P(N), a \in P(N)$. Since $T^{*}$ eliminates quantifiers, $a \in \operatorname{acl}^{*}(B)$ if and only if there is an $\mathcal{L}$-formula $\varphi(x, \vec{y}, \vec{z})$ and tuples $\vec{m} \in M, \vec{b} \in B$ such that $M=\varphi(a, \vec{b}, \vec{m})$ and the formula $\varphi(x, \vec{b}, \vec{m})$ has finitely many realizations in $P(N)$. The last condition is equivalent to $\varphi(x, \vec{b}, \vec{m})$ being algebraic in $T$. It follows that for any $B \subset P(N), \operatorname{acl}^{*}(B)=$ $\operatorname{acl}_{M}(B) \cap P(N)$, and since $\operatorname{acl}_{M}$ satisfies the exchange property in $N$, same is true for acl*.

To show that $T^{*}$ eliminates $\exists^{\infty}$, consider any $\mathcal{L}^{*}$-formula $\psi(x, \vec{y})$. By quantifier elimination in $T^{*}$, it is equivalent to an $\mathcal{L}$-formula $\varphi(x, \vec{y}, \vec{m})$ with parameters $\vec{m} \in$ $M$. For any $\vec{b} \in P(N), \psi(x, \vec{b})$ is algebraic if and only if $\varphi(x, \vec{b}, \vec{m})$ has finitely many solutions in $P(N)$, which is equivalent to algebraicity of $\varphi(x, \vec{b}, \vec{m})$ in $N$. Since $T$ eliminates $\exists^{\infty}, \varphi(x, \vec{b}, \vec{m})$ is algebraic if and only if $N \models \theta(\vec{b}, \vec{m})$ for some $\mathcal{L}$-formula $\theta(\vec{y}, \vec{z})$. Let $R_{\theta}(\vec{y})$ correspond to $\theta(\vec{y}, \vec{m})$. Thus for any $\vec{b} \in P(N), \psi(x, \vec{b})$ is algebraic if and only if $R_{\theta}(\vec{b})$ holds in $P(N)$ viewed as a model of $T^{*}$, as needed.

Since $T^{*}$ is again geometric, $T^{*}$ has a notion of independence induced by acl* As before, we let $(N, P)$ be a saturated model of $T h_{M}(M, P)$. For $A, B, C \subset P(N)$ sets, we write $A \downarrow_{B}^{*} C$ to mean that $A$ is acl ${ }^{*}$-independent from $C$ over $B$. Note that by [22, Theorem 2.3] when $T$ is simple of $S U$-rank one, our notion of independence coincides with non-forking in $T^{*}$.

Lemma 5.6. Assume that $T$ is a geometric theory which is weakly 1-based. Then $T^{*}$ is weakly 1-based.

Proof. Let $(N, P)$ be a saturated model of $T h_{M}(M, P)$. Let $\vec{a} \in P(N)$ and let $B \subset P(N)$ be a set. By hypothesis there is a superset $C$ of $B M$ such that $\vec{a} \downarrow_{B M} C$ and whenever $\vec{a}^{\prime} \models \operatorname{tp}(\vec{a} / C)$ is independent from $\vec{a}$ over $C$, we have $\vec{a} \downarrow_{\vec{a}^{\prime}} B M$. Since $\operatorname{acl}^{*}=\operatorname{acl}_{M}, C$ is a witness for the desired property in $T^{*}$.

5.3. Fields expanded with a group having the Mann property. In this section we deal with the theory of a dense divisible multiplicative subgroup with the Mann property of a real closed field $K$ as presented by van den Dries and Günaydin in [11]. These structures are analyzed by adding a predicate $G$ to the real closed field, where $G$ is interpreted as the multiplicative group and considering the new structure $(K, G)$. A description of definable sets of $K$ and of $G$ in such a structure can be found in [11]. It was proved by Berenstein, Ealy and Günaydin [4] that such a pair $(K, G)$ is super-rosy of p-rank $\omega$ and that p-rank $(G)=1$ (seen as a 
definable subset of the pair). In particular, $G$ as a subset of the structure $(K, G)$ is a pregeometry. Our goal is to show that the theory of $G$ with the induced structure is weakly 1 -based.

We proceed as in the previous subsection. For each $\mathcal{L}$-formula $\varphi(x)$ with parameters in $K$, we introduce a new predicate symbol $R_{\varphi}$. Let $\mathcal{L}^{*}$ be the resulting language. We denote by $K^{*}$ the structure $K$ with the natural interpretation for the new relations and $G^{*}$ the substructure with universe $G$. Finally let $T^{*}$ be the theory of $G^{*}$, it is important to note that the theory $T^{*}$ depends on the underlying field $K$. We denote the algebraic closure in models if $T$ by acl and in models of $T^{*}$ by acl $^{*}$.

As in the previous section it can be proved that $T^{*}$ has quantifier elimination and that $\operatorname{acl}(-\cup K)=\operatorname{acl}^{*}(-)$. In particular $T^{*}$ is a geometric theory.

Our work depends on the following facts:

Fact 5.7 (Theorem 7.2 [11]). Let $K$ be a real closed field and let $G$ be a dense divisible multiplicative subgroup of $K^{>0}$ having the Mann property. Then if $X \subset$ $G^{n}$ is definable, there is $Y \subset K^{n}$ definable in $K$ (seen as an ordered field) such $X=Y \cap G^{n}$.

This fact remains true in a saturated model of $T h_{K}(K, G)$, since we only added new constants to the language.

From the previous fact it easily follows that if $\vec{a} \in G^{*}, B \subset K \cup G^{*}$ and $\operatorname{dim}(\vec{a} / B)<\operatorname{dim}(\vec{a})$, then there is a polynomial $f(\vec{y}) \in \mathbb{Q}(B)[y]$ such that $f(\vec{a})=0$. In particular, we need to understand the solutions of algebraic varieties in $G^{*}$. This is characterized in [11]

Definition 5.8. For any $n$-tuple $k=\left(k_{1}, \ldots, k_{n}\right) \in \mathbb{Z}^{n}$ consider the character $\chi_{k}$ : $\left(K^{\times}\right)^{n} \rightarrow K^{\times}$given by $\chi_{k}\left(x_{1}, \ldots, x_{n}\right)=x_{1}^{k_{1}} \cdots x_{n}^{k_{n}}$. We let $\mathcal{D}(n, d)$ be the finite collection of subgroups of $\left(K^{\times}\right)^{n}$ that are the intersection of kernels of characters $\chi_{k}$ with $|k|=\left|k_{1}\right|+\cdots+\left|k_{n}\right| \leq d$.

Proposition 5.9. Let $f_{1}, \ldots, f_{m} \in K\left[X_{1}, \ldots, X_{n}\right]$ have degree $\leq d$, and let $V=$ $\left\{x \in K^{n}: f_{1}(x)=\cdots=f_{m}(x)=0\right\}$. Suppose $G$ has the Mann property. Then $V \cap G^{n}$ is a finite union of cosets of subgroups $D \cap G^{n}$ of $G^{n}$ with $D \in \mathcal{D}(n, d)$.

Proof. This proposition is proved in [11, Proposition 5.8] when $K$ is an algebraically closed field. The same proof, that only depends on the Mann property, holds when $K$ is a real closed field.

The conclusion of the proposition is also true for a saturated model of $T h_{K}(K, G)$ since the statement is an elementary property.

Proposition 5.10. Let $K$ be a real closed field and let $G$ be a dense divisible multiplicative subgroup of $K^{>0}$ having the Mann property. Then the theory of $G^{*}$ is weakly 1-based.

Proof. We work in a saturated model $\left(K^{*}, G^{*}\right)$ of $T h_{K}(K, G)$ in the language $\mathcal{L}^{*}$. Assume as above that $\vec{a} \in G^{*}, B \subset K \cup G^{*}$ and $\operatorname{dim}(\vec{a} / B)<\operatorname{dim}(\vec{a})$. Let $V$ be a variety of dimension $\operatorname{dim}(\vec{a} / B)$ definable over $B$ such that $\vec{a} \in V$. Then $V \cap\left(G^{*}\right)^{n}$ is equivalent to a disjunction $\vee_{i \leq t} \vec{c}_{i}\left(D_{i} \cap\left(G^{*}\right)^{n}\right)$, where each $D_{i}$ is the intersection of kernels of characters and thus $D_{i} \cap\left(G^{*}\right)^{n}$ is a $\emptyset$-definable subgroup of $\left(G^{*}\right)^{n}$. Assume $D_{i}$ is the kernel of the characters $\chi_{i j}\left(x_{1}, \ldots, x_{n}\right), j \leq m_{i}$ and that $\vec{c}_{i}=\left(c_{i 1}, \ldots, c_{i n}\right)$. Then $\chi_{i j}\left(a_{1}, \ldots, a_{n}\right)=\chi_{i j}\left(c_{i 1}, \ldots, c_{i n}\right)$ so we may assume 
that $\vec{c}_{i} \in\left(G^{*}\right)^{n}$. After taking a non thorn-forking extension of $\operatorname{tp}_{*}\left(\vec{c}_{1}, \ldots, \vec{c}_{t} / B\right)$ we may further assume that $\vec{c}_{1}, \ldots, \vec{c}_{t}$ are free from $a_{1}, \ldots, a_{n}$ over $B$.

Let $C=B \cup\left\{\vec{c}_{1}, \ldots, \vec{c}_{t}\right\}$ and let $\left(a_{1}^{\prime}, \ldots, a_{n}^{\prime}\right) \models \operatorname{tp}\left(a_{1}, \ldots, a_{n} / C\right)$ be such that $\left(a_{1}^{\prime}, \ldots, a_{n}^{\prime}\right) \downarrow_{C}^{\mathrm{b}}\left(a_{1}, \ldots, a_{n}\right)$. Then we have $\chi_{j i}\left(a_{1}, \ldots, a_{n}\right)=\chi_{j i}\left(c_{i 1}, \ldots, c_{i n}\right)=$ $\chi_{j i}\left(a_{1}^{\prime}, \ldots, a_{n}^{\prime}\right)$ for some $i \leq t$ and all $j \leq m_{i}$, so $\vec{c}_{i}\left(D_{i} \cap\left(G^{*}\right)^{n}\right)$ is definable over $\left\{a_{1}^{\prime}, \ldots, a_{n}^{\prime}\right\}$ and $\operatorname{dim}\left(a_{1}, \ldots, a_{n} / a_{1}^{\prime}, \ldots, a_{n}^{\prime}\right) \leq \operatorname{dim}\left(a_{1}, \ldots, a_{n} / C\right)=\operatorname{dim}\left(a_{1}, \ldots, a_{n} / B\right)$. In particular, $\left(a_{1}, \ldots, a_{n}\right) \downarrow_{\left(a_{1}^{\prime}, \ldots, a_{n}^{\prime}\right)}^{\mathrm{b}} B$.

\section{LOVELY PAIRS AND DENSE EMBEDDINGS}

In this section we relate the notion of lovely pairs of geometric structures to that of dense embeddings developed by Macintyre in [16]. We will review some of the notions introduced in [16] and prove that for the geometric theories $T$ considered in [16], Macintyre's theory $T^{d}$ of dense embeddings of models of $T$ coincides with the theory $T_{P}$ of lovely pairs of models of $T$.

We start with reviewing some definitions. Let $T$ be a pregeometric theory.

Definition 6.1. Let $N, M=T$ with $N \preceq M, N \neq M$. We say that $(M, N)$ is a Vaughtian pair if for some formula $\varphi(x, \vec{a})$ with parameters $\vec{a} \in N$ with infinitely many solutions in $N$ we have $\varphi(N)=\varphi(M)$. We say that $T$ has a Vaughtian pair if there are $N, M \models T$ such that $(M, N)$ is a Vaughtian pair.

Lemma 6.2. Let $(M, P) \models T_{P}$. Then $(M, P(M)$ is not a Vaughtian pair.

Proof. Let $\varphi(x, \vec{a})$ be an -formula with parameters in $P(M)$ with infinitely many solutions, so $P(M) \models \exists^{\infty} x \varphi(x, \vec{a})$. Let $\left(M^{\prime}, P\right) \succeq(M, P)$ be saturated, so $\left(M^{\prime}, P\right)$ is a lovely pair of models of $T$. Let $p(x)$ be a complete non-algebraic type over $\vec{a}$ containing $\varphi(x, \vec{a})$. Since $\left(M^{\prime}, P\right)$ is a lovely pair, there is a realization $b$ of $p(x)$ in $M^{\prime}$ which is free from $P\left(M^{\prime}\right)$. In particular, $b \in \varphi\left(M^{\prime}\right) \backslash \varphi\left(P\left(M^{\prime}\right)\right)$, so $\left(M^{\prime}, P\right) \models$ $\exists x \varphi(x, \vec{a}) \wedge \neg P(x)$. Thus $(M, P) \models \exists x \varphi(x, \vec{a}) \wedge \neg P(x)$ and $\varphi(M, \vec{a}) \neq \varphi(P(M), \vec{a})$ as we wanted.

Thus, the class of models that we consider when dealing with lovely pairs are not Vaughtian pairs, but the underlying theory $T$ under consideration may have Vaughtian pairs as shown by the following example:

Example 6.3. Consider the theory DLO of dense linear orders without endpoints. Let $M=\mathbb{R}$ and let $N=(\mathbb{R} \cap(-\infty, 0]) \cup \mathbb{Q}^{+}$. Then $(M, N)$ is a Vaughtian pair

Fact 6.4. Assume that $T$ does not have Vaughtian pairs. Then $T$ eliminates the quantifiers $\exists^{\infty}$.

Proof. See Lemma 5 in [16].

The pregeometric theories $T$ considered in [16] do not have Vaughtian pairs. First of all this implies that under this extra assumption $T$ is geometric, so the tools from lovely pairs developed in [5] apply. On the other hand the example above shows that the family of theories under consideration in [16] is strickly smaller than the class of geometric theories.

The notion of dense pairs in [16] is word by word the notion that we call in Definition 1.2 the density/coheir property. In order to conclude that the dense embeddings are lovely pairs, we need to show that the extension property holds in saturated models of dense embeddings. 
Fact 6.5. Suppose $T$ satisfies the assumptions $1-6$ listed in [16] and let $(M, N) \models$ $T^{d}$. Suppose that card $(M)=\operatorname{dim}(M / N)=\operatorname{dim}(N) \geq|\mathcal{L}|$. Then there is a basis $X$ of $M$ over $N$ and a basis $Y$ of $N$ such that for every infinite definable set $D$ over $M, X \cap D \neq \emptyset$ and $Y \cap D \neq \emptyset$.

Proof. See Lemma 8 in [16].

Lemma 6.6. Suppose $T$ satisfies the assumptions $1-6$ listed in [16]. Let $(M, N)$ be a saturated model of $T^{d}$. Let $\vec{m}$ be a tuple of elements in $M$ and let $\varphi(x, \vec{m})$ be an $\mathcal{L}$-formula with infinitely many realizations. Then there is a realization of $\varphi(x, \vec{m})$ in $M$ which is free from $\vec{m} \cup N$.

Proof. Let $X$ be as in the previous fact. Let $X_{0} \subset X$ finite and $Y_{0} \subset Y$ finite such that $\vec{m} \subset \operatorname{acl}\left(X_{0} \cup Y_{0}\right)$. Let $\psi\left(x, \vec{m}, X_{0}, Y_{0}\right)=\varphi(x, \vec{m}) \wedge_{y \in X_{0} \cup Y_{0}}(x \neq y)$. By the fact there is an $a \in X$ satisfying $\psi$. Since $X$ is a basis of $M$ over $N, a \notin \operatorname{acl}\left(X_{0}, N\right)$ and $M \models \varphi(a, \vec{m})$ as we wanted.

Proposition 6.7. Suppose $T$ satisfies the assumptions $1-6$ listed in [16]. Let $(M, N)$ be a saturated model of $T^{d}$. Then $(M, N)$ is a lovely pair of models of $T$.

Proof. As pointed out earlier, such theories $T$ are geometric. The assumption that $P(M)$ is dense in $M$ translates to the coheir property. Finally the previous lemma implies the extension property.

\section{REFERENCES}

[1] H. AdLER, A geometric introduction to forking and thorn-forking, preprint.

[2] Y. Baisalov and B. Poizat, Paires de structures o-minimales, Journal of Symbolic Logic, 63 (1998), 570-578.

[3] I. Ben YaAcov, A. Pillay, E. Vassiliev, Lovely pairs of models, Annals of pure and applied logic 122 (2003), pp. 235-261.

[4] A. Berenstein, C. Ealy, A. Günaydin, Thorn independence in the field of real numbers with a small multiplicative group, Annals of Pure and Applied Logic. Volume 150, Issues 1-3, December 2007, pp. 1-18.

5] A. Berenstein and E. Vassiliev, On lovely pairs of geometric structures, Annals of Pure and Applied Logic, Volume 161, Issue 7, April 2010, pp. 866-878.

[6] G Boxall, Lovely pairs of models of a p-rank 1 theory, preprint.

[7] S. Buechler, Pseudoprojective Strongly Minimal Sets are Locally Projective, Journal of Symbolic Logic, 56, (1991), pp. 1184-1194

[8] Z. Chatzidakis and A. Pillay, Generic structures and simple theories, Annals of Pure and Applied Logic, (1998), pp. 71-92.

[9] T. De Piro, B. Kim, The geometry of 1-based minimal types, Trans. Amer. Math. Soc. 355 (2003), 4241-4263.

[10] L. VAN DEN DRIES, Dense pairs of o-minimal structures, Fund. Math. 157 (1998), pp. 61-78.

[11] L. van den Dries, A. Günaydin The fields of real and complex numbers with a small multiplicative group, Proc. London Math. Soc. (3) 93 (2006), no. 1, 43-81.

[12] C. Ealy and A. Onshuus, Characterizing Rosy Theories, J. Symbolic Logic Volume 72, Issue 3 (2007), 919-940.

[13] J. Gagelman, Stability in geometric theories, Annals of Pure and Applied Logic 132 (2005), 313-326.

[14] E. Hrushovski and A. Pillay, Groups definable in local fields and psedofinite fields, Israel Journal of Mathematics 85 (1994), no. 1-3, 203-262.

[15] J. Loveys and Y. Peterzil, Linear o-minimal structures, Israel Journal of Mathematics 81 (1993), 1-30.

[16] A. Macintyre, Dense embeddings I: A Theorem of Robinson in a general setting, in Model Theory and Algebra, a memorial tribute to Abraham Robinson, Lecture Notes in Mathematics 498,1975 , pp. 200-219. 
[17] A. Onshuus, Properties and consequences of thorn-independence, Journal of Symbolic Logic, 71 (2006), pp 1-21.

[18] Y. Peterzil and S. Starchenko, A trichotomy theorem for o-minimal structures, Proceedings of the London Mathematical Society, (2000), pp. 481-523.

[19] Y. Peterzil and S. Starchenko, Geometry, Calculus and Zil'ber's conjecture, The bulletin of symbolic logic, vol 2, no 1, (1996), pp. 72-83.

[20] A. Pillay, Geometric Stability Theory, Oxford Logic Guides 32, Clarendon Press, Oxford, 1996.

[21] A. Pillay, Canonical bases in o-minimal and related structures, preprint 2006.

[22] A. Pillay and E. Vassiliev, On lovely pairs and the $\exists y \in P$ quantifier, Notre Dame Journal of Formal Logic, 46 (2005), 491-501.

[23] E. Vassiliev, Generic pairs of SU-rank 1 structures, Annals of Pure and Applied Logic, 120, (2003), pp. 103-149.

Universidad de los Andes, Cra 1 No 18A-10, Bogotá, Colombia

E-mail address: aberenst@uniandes.edu.co

$U R L$ : www . matematicas . uniandes . edu.co/ aberenst

Sir Wilfred Grenfell College, Memorial University of Newfoundland, Corner Brook, NL A2H 6P9, Canada

E-mail address: yvasilyev@swgc.mun.ca 